\title{
MAC Protocol with Regression based Dynamic Duty Cycle Feature for Mission Critical Applications in WSN
}

\author{
Gayatri Sakya \\ Department of Electronics and Communication Engineering \\ JSS Academy of Technical Education, \\ NOIDA, U.P., India
}

\begin{abstract}
Wireless sensor networks demand energy efficient and application specific medium access control protocol when deployed in critical areas which are not frequently accessible. In such areas, the residual energy of nodes also become important along with the efficient data delivery. Many techniques using adaptive duty cycle approach are suggested by researchers to improve the data delivery performance of protocols. As low duty cycle introduces delay and high duty cycle causes energy losses in the network so duty cycle adaptation according to the distribution of nodes near event occurring area, traffic behaviour and remaining energy of the nodes may be done for energy saving as well as efficient data delivery performance. After analysing the S-MAC protocol performance in critical scenarios for the residual energy, throughput and packet delivery ratio, this paper suggests an improved mission critical MAC protocol called MC-MAC which uses novel regression based adaptive duty cycle approach. The duty cycle is given by the regression pattern of traffic while considering the performance of SMAC protocol for residual energy, throughput and packet delivery ratio. The analytical model of MC-MAC protocol is given accordingly and the performance analysis shows that the proposed MC-MAC protocol saves $40 \%$ energy of whole network and also $20 \%$ energy of the critical nodes in the mission critical path till base station, as compared to SMAC protocol. Very few improved MAC protocols provide mechanism to save the residual energy of critical nodes and hence to improve the lifetime of critical path. As MC-MAC protocol considers the throughput and packets delivery ratio (also along with residual energy) for calculating the regression formula for duty cycle based on traffic, so it is better than other critical MAC protocols which does trade-off of energy with throughput and packet delivery ratio.
\end{abstract}

Keywords-Regression based adaptive duty cycle approach; mission critical MAC; analytical model; performance analysis

\section{INTRODUCTION}

The wireless sensor networks consists of sensor nodes deployed in large numbers to gather information and send it to base station for further actions. These nodes consist of microcontrollers, sensors and transceivers and are battery operated as shown in Fig. 1.

The nodes are battery powered so power saving is the biggest challenge to researchers in wireless sensor networks for increasing lifetime of the network once the nodes are deployed.

\author{
Dr. Vidushi Sharma \\ Department of Information and Communication Technology \\ Gautam Buddha University, \\ Greater Noida, U.P., India
}

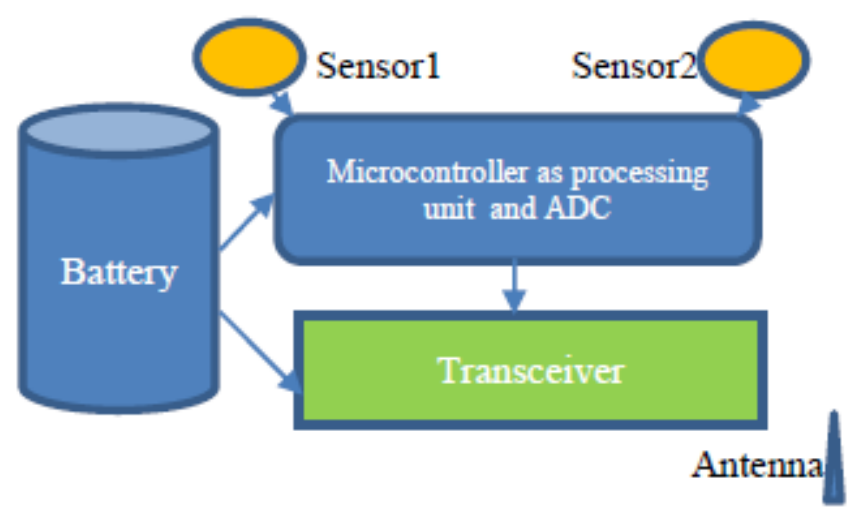

Fig. 1. Architecture of wireless sensor node.

The nodes are battery operated and most of the energy is consumed by the transceiver of the node in communication [1]. Nowadays wireless sensor networks are widely used in some critical applications like gas leakage detection, surveillance of border, patients monitoring system, monitoring volcanic activities, tsunami alert system, etc. Wireless sensor nodes which are deployed once, need to stay active for years to gather information which they sends to base stations. So apart from the data delivery performance, the residual energy of nodes plays an important role to keep the network path alive till base station nodes. The protocol stack for wireless sensor networks is not standardized because of the infinite applications of sensor nodes in almost all the fields of engineering and sciences. According to diversified applications of sensor networks, the existing protocols at MAC layer need to be designed differently which can meet the demand of fast response in mission critical areas along with energy saving. In mission critical applications, traffic load increases suddenly on nodes near event occurring area and they need to send information to base station in timely and reliable fashion. Several MAC protocols were proposed based on the existing basic IEEE 802.11 MAC protocol for wireless LAN [1]. Some protocols introduced periodic listing mechanism, some changed the contention mechanism and some protocols introduced adaptive listening mechanism to make it suitable for wireless sensor networks. But very few protocols talked about the MAC protocol suitable for critical applications of wireless 
sensor network which is the need of the hour. The Sensor MAC (SMAC) [2] protocol is the most popular contention based wireless sensor network MAC protocol which introduced the periodic listen and sleep mechanism to save energy of the node. This protocol is already implemented and tested on hardware. The Sensor MAC protocol is an improvement of 802.11 MAC with sleep/awake mechanism to avoid the idle listening problem in 802.11 MAC protocol. The sensor MAC protocol performs well in slow traffic but in high traffic introduces sleep delay because of its periodic sleeping mechanism. Many improvements are done on Sensor MAC protocol to improve its performance but very few researchers have studied the Sensor MAC protocol performance in mission critical scenarios. This motivated to test the performance of SMAC protocol for mission critical applications and then to propose a new model for the mission critical MAC protocol. Medium access control protocols can save energy by intelligently using the transceiver power using periodic sleeping when there is no traffic, by avoiding collision and retransmission of packets and also by avoiding control packet overhead using one RTS/CTS pair to send data.

Mission critical applications are applications running in volcanic areas, in oceans or surveillance applications on border where quick response is required. These areas are not accessible frequently or instantly so the nodes cannot be replaced easily. The lifetime of network and the data delivery performance without delay is utmost importance So the residual energy of the sensor nodes and hence network lifetime is very essential .This paper analyses the Sensor MAC protocol for mission critical scenarios and based on these results proposes a new MC-MAC protocol model suitable for mission critical scenarios. Toward this goal, a novel approach based on regression is used for making $\mathrm{MC}-\mathrm{MAC}$ protocol adaptive to the traffic scenarios while considering the residual energy of nodes and other mission critical performance parameters.

The paper is divided into various sections. In Section 2, the literature review and motivation for designing MC-MAC protocol is discussed. Section 3 represents the MC-MAC protocol model methodology. Section 4 gives the analytical model and performance analysis of the proposed protocol model and compares it with the basic Sensor MAC protocol. Section 5 discusses the conclusion of the work and Section 6 finally ends with the future scope of the work.

\section{RELATED WORK}

\section{Literature Review and Motivation}

To develop a new protocol, the fundamental contention based Sensor MAC protocol is critically analyzed and thereafter its improvements are also reviewed which motivated to design a new model of mission critical MAC protocol.

\section{A. Sensor MAC protocol critical analysis}

S-MAC [2] protocol uses periodic listen and sleep mechanism for increasing the lifetime of the node. The frame interval is divided into listen time and sleep time as shown in Fig. 1.The nodes periodically wake up and the go to sleep to avoid the idle listening. During the sleep period node turns off its transceiver which saves maximum amount of energy. The listen period is fixed in SMAC and the duty cycle is controllable parameter varying from $1 \%$ to $100 \%$. The improvements on 802.11 MAC is done to avoid idle listening using periodic listen-sleep of nodes, to avoid overhearing by turning off the transceiver after hearing RTS/CTS packets destined for other node and control packets overhead reduction by using single pair of RTS/CTS packet for transmission of data available. The duty cycle of the Sensor-MAC protocol is fixed during the initial deployment and is not changed when the traffic load and residual energy of the node changes. This makes it unsuitable for mission critical applications where high traffic rate signifies some important information to be delivered and immediate response is sought. So to minimize sleep delay the value of duty cycle should be increased, keeping constraint of residual energy of the node.



Fig. 2. SMAC frame.

The SMAC frame time is the sum of listen time and sleep time as shown in Fig. 2 and hence the energy saving in SMAC with periodic sleep is directly proportional to sleep time. The duty cycle of S-MAC is given by (1).

$$
\text { Duty cycle }(\mathrm{d})=(\text { Listen Time }) /(\text { Listen Time }+ \text { Sleep Time })
$$

Now from (1), it's implied that at low duty cycle, the sleep time in a frame will be relatively more and hence the energy will be saved. The future directions from [2] are also concluded as to limit the sleep period for decreasing the latency. The energy consumption in intermediate nodes is more because of SYNC overhead. Also the border nodes can follow multiple schedules, so they have to wakeup multiple times which consumes more energy. The Sensor MAC protocol uses message passing technique which should provide a limit on the number of times the extensions is given for the lost frames, in case of dead receiver.

\section{B. Improved MAC protocols based on adaptive duty cycle approach.}

Wei Ye, et al. in 2004 suggested an improvement on Sensor MAC protocol which used coordinated adaptive listening of the nodes to improve the latency [3]. In this protocol the neighboring nodes who overhears RTS and CTS packets wakeup for a short period of time after transmission. So if the node is next hop node, its neighbor will be able to pass data to it instead of waiting for the scheduled wakeup time. The drawback is that all the neighboring nodes who overhear RTS/CTS packets will awake for short period, which results in energy consumption. So latency is improved at the cost of residual energy of nodes.

In 2004, P. Lin, et al. proposed DSMAC protocol [4]. DSMAC protocol also tune the duty cycle of nodes according to the delay and residual energy of the node. The multiple duty cycle concept is used in DSMAC to improve the latency but at the cost of energy consumption, with not much improvement in 
energy seen at high traffic rates. The mission critical scenarios are not taken care where suddenly the traffic rate increases on occurrence of an event.

In 2005, [5] Yang, et al. suggested utilization based tuning of duty cycle in their protocol. In U-MAC the duty cycle of the nodes are tuned based on the utilization function which calculated the load on each node. In U-MAC the forwarding node will always have the more utilization and hence all-time its duty cycle will be higher so soon it will die out even at low traffic loads. Hence, U-MAC protocol does not provide solution to improve the network lifetime in mission critical scenarios.

In 2006 Demirkol, et al. discussed the sensor network properties which should be taken care while designing the MAC protocols [6]. The authors discussed various many other existing MAC protocols which include contention based and contention free like TDMA approach also. TDMA approach has the feature of collision free access to channel but the network is not adaptable to topology changes when new nodes inserted or existing node dies out. Because of short range, the wireless sensor networks are dense networks. The main challenge of wireless sensor network is energy efficient sharing of communication channel. Multiple nodes in the same region want to transmit information to other nodes. The channel access mechanism thus primarily classified the medium access protocols as contention based, contention free and hybrid which use both the concepts.

Another challenge is confronted when wireless sensor nodes are used in critical application in last few years. Along with the energy efficiency, the throughput and packet loss rate also considered as important parameters for protocol performance in critical scenarios. In wireless sensor network applications, nodes play different roles like source nodes, intermediate nodes and the sink nodes. According to the location of event the role of the nodes changes in network. So if the role of nodes changes according to the scenarios, fixed sleep-listen schedule as in S-MAC will result in energy waste in nodes when no data packets to be forwarded by the nodes and also increase the buffer size on the nodes which are taking part in transmission. So further this work concentered on the existing protocols which used adaptive duty cycle approach.

In 2010, Mishra, et al. tuned the slot time in the contention window in listen period for adaptive listening but used a complex fuzzy based approach [7]. As the nodes have very less storage capacity so two fuzzy algorithms used in the paper may be replaced by some simple techniques.

In 2012, Suriyanchai, et al. [8] did the study and classification of MAC protocols on the basis of reliability and timely data delivery performance. The mission critical MAC protocol performance parameters based on reliability can be the packet loss rate and packet delivery ratio and the throughput signifies the data delivery in time.

In 2012, Hsu, Tz-Heng, et al. in [9] also gave the dynamic traffic aware MAC protocol based on tuning the duty cycle, they concentrated more on the data transmission rate and latency but the energy consumption is extremely increased in their protocol, which is not suitable for the mission critical case.

In 2013, Sakya G, et al. performed the analysis of SMAC protocol for single hop scenario in ns 2.35 [10].

In 2013, G. Sakya, et al. studied the popular SMAC in ns2.35 for various packet arrival rates at different values of duty cycles in multi-hop scenario [11] also. The SMAC protocol is tested from low traffic rates to very high traffic rates in multihop scenario. It is observed that the residual energy reaches maximum if the duty cycle is $20 \%$ under high traffic rates $(.01 \mathrm{~s}$ to $.1 \mathrm{~s}$ packet inter arrival time) along with the optimum performance of packet delivery ratio and throughput. The authors concluded that under mission critical scenarios if the value of duty cycle is made high, then the residual energy can be saved as compared to low duty cycle. Tuning of duty cycle with respect to certain parameters in MAC protocol is the technique which may be used to improve the residual energy, throughput and packet delivery ratio of the network and also of the individual nodes.

In 2013, adaptive duty cycle control is also done by Byun, et al. They proposed the tuning of sleep time based on the queue management feedback control system to achieve efficient performance in heavy traffic [12].

In 2015, Donghong $\mathrm{Xu}$ and $\mathrm{Ke}$ Wang, et al. in proposed EA-MAC, an adaptive traffic aware MAC protocol based on correlation of nodes [13]. They also made duty cycle adaptive based on the bases of predicted flow on the node. The protocol performance in terms of energy consumption is very similar to S-MAC protocol, so in spite of having better throughput, delay and packet loss rate the protocol is not suitable for applications in which nodes cannot be replaced frequently, once deployed.

In 2016, G. Sakya, et al. analyzed the performance of SMAC in grid scenario and suggested improvements on SMAC to make it suitable for critical applications in grid scenario [14].

To develop the required mission critical MAC protocol, this paper analyzes the performance of S-MAC protocol for residual energy, throughput and packet delivery ratio and studied the data pattern and its behavior. Differing from other proposed protocol, it proposes to tune the duty cycle based on the output parameters like residual energy, throughput and packet delivery ratio under different traffic scenarios using novel regression technique. The analytical model is prepared for performance analysis of the proposed model. The proposed protocol is designed with the following objectives:

1) To save the residual energy of the network, the duty cycle of only selected nodes in event occurring area with high traffic is made high. It takes into consideration the residual energy of node also.

2) Duty cycle allocation is based on regressive analysis of Sensor MAC protocol performance parameters throughput, packet delivery ratio and residual energy with respect to traffic and duty cycle. This is done to improve the overall performance of the protocol. 
3) Analytical model is developed to show the effect of this novel duty cycle adaptation approach on the residual energy saving of mission critical MC-MAC protocol.

\section{MC-MAC PRotocol Design}

MC-MAC protocol is based on the novel approach where the results obtained from the S-MAC protocol analysis in mission critical applications for residual energy, throughput and packet delivery ratio are used to make the duty cycle adaptive. The duty cycle is made adaptive according to the observed regressive behavior of it based on traffic while considering the performance of the mission critical parameters for residual energy, throughput and packet delivery ratio.

This work consider first a multi-hop scenario of consists of 11 nodes with one source and one sink as shown in Fig. 3. The node 0 (source node) generates the packets at different time interval in the range $.01 \mathrm{~s}$ to $50 \mathrm{~s}$. All nodes have initial energy of 1000 joules. The performance of SMAC protocol is tested in ns-2.35. The nodes are places $200 \mathrm{~m}$ apart and the routing protocol used is DSR. The CBR packets of size 80 bytes are used for data transmission. The queue size is taken as maximum 50 packets. The simulation runs for 10,000 second each time. The data generated in the trace file is used to summarize the performance of the protocol. The parameters considered in energy model are based on [14], [15].

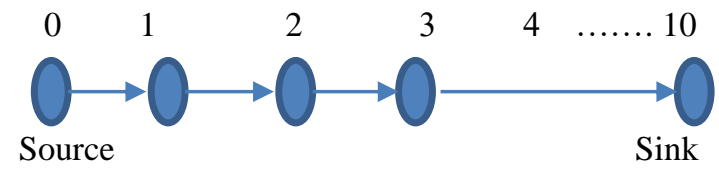

Fig. 3. Network topology

The duty cycle values are varies and the performance of SMAC protocol is tested under different scenarios. The impact of changing the duty cycle according to the traffic loads is observed and presented in Table 1.

TABLE. I. SMAC PRotocol PERFormance

\begin{tabular}{|l|l|l|l|l|}
\hline $\begin{array}{l}\text { Duty } \\
\text { cycle \% }\end{array}$ & $\begin{array}{l}\text { Packet } \\
\text { inter arrival } \\
\text { time } \\
\text { (seconds) }\end{array}$ & $\begin{array}{l}\text { Residual } \\
\text { energy in } \\
\text { (Joules) }\end{array}$ & $\begin{array}{l}\text { Packet } \\
\text { delivery } \\
\text { ratio (\%) }\end{array}$ & $\begin{array}{l}\text { Throughput } \\
\text { (Kbps) }\end{array}$ \\
\hline 20 & .1 & 1304.286 & 60.4222 & 0.13 \\
\hline 30 & .1 & 1048.182 & 57.9378 & 0.2 \\
\hline 40 & .1 & 688.1242 & 84.8014 & 0.28 \\
\hline 20 & 1 & 1292.846 & 52.2422 & 0.06 \\
\hline 30 & 1 & 1102.84 & 54.6851 & 0.06 \\
\hline 40 & 1 & 789.1993 & 57.3691 & 0.06 \\
\hline 20 & 10 & 1002.848 & 84.3318 & 0.06 \\
\hline 30 & 10 & 550.9717 & 57.9378 & 0.06 \\
\hline 40 & 10 & 368.9273 & 94.7644 & 0.06 \\
\hline 20 & 20 & 751.0417 & 89.8551 & .03 \\
\hline 30 & 20 & 386.1013 & 95.0413 & 0.03 \\
\hline 40 & 20 & 240.8189 & 97.0874 & 0.03 \\
\hline
\end{tabular}

It has been observed in Table 1 that under high traffic rates, for efficient throughput and packet delivery ratio, the duty cycle must be $40 \%$. But along with that to make the protocol energy efficient we have to take care of the energy loss because of this high duty cycle. So in mission critical scenarios, where the traffic rate increases suddenly, we should choose high value of duty cycle for efficient data transmission. In order to save energy, in medium traffic loads, we can choose the duty cycle value as $20 \%$, to have efficient data transmission.

Also, it has been observed that at larger packet inter arrival time at 40 s, the energy saving is 1151.221 joules and also the throughput and packet delivery ratio is .01 kbps and $82.5 \%$ at $10 \%$ duty cycle.

As regression is a measure of average relationship between two or more variables in terms original units of data so we applied this analysis to choose the duty cycle in different scenarios in the wireless sensor networks. The above data is summarized and the following regression expression for duty cycle factor is obtained in Table 2 .

From the results obtained from Table 2, the relation between the packet arrivals rate (packets/second) and the duty cycle is obtained as follows:

Duty cycle factor $=(.20953116) *$ traffic $($ packets/second $)+$ 20.83677

The duty cycle will be modified in the synchronization module of the protocol which modifies the synchronization packet and sends the synchronization packet to its neighbors at the next synchronization period. The synchronization packet incudes the information about the traffic rate of the node. The duty cycle allocation to the nodes in the network can be done based on this formula calculated. The duty cycle of SMAC protocol can be allocated dynamically to the nodes based on this expression, which takes care of all the output parameters like residual energy, throughput and packet delivery ratio. The sudden increase in the traffic of certain nodes occurs when they send data frequently on the occurrence of event. So according to the traffic only selected nodes tune their duty cycle and send the updated schedule in the synchronization packet to their neighbors.

To minimize the synchronization problem the algorithm uses duty cycle values only $10 \%, 20 \%$ and $40 \%$. So the changed duty cycle will not affect the communication among nodes whose duty cycle is unaffected.

The duty cycle factor is calculated dynamically using (2).

1) If (Duty cycle factor $\geq 30$ ) then assign duty cycle as $40 \%$.

2) If $(15 \leq$ Duty cycle factor $\leq 30)$ then assign duty cycle as $20 \%$.

3) If (Duty cycle factor $<15$ ) then assign duty cycle as $10 \%$. Since the base duty cycle is $10 \%$. 
TABLE. II. REGRESSION ANALYSIS OF SMAC PROTOCOL

\begin{tabular}{|l|l|l|l|l|l|l|l|l|}
\hline & Coefficients & $\begin{array}{l}\text { Standard } \\
\text { Error }\end{array}$ & t Stat & P-value & Lower 95\% & Upper 95\% & Lower 95.0\% \\
\hline Intercept & 20.8367754 & 3.879835 & 5.370531 & 0.001711 & 11.34316 & 30.33039 & 11.34316 \\
\hline packets/s & 0.209531116 & 0.109188 & 1.91899 & 0.103411 & -0.05764 & 0.476705 & -0.05764 & 30.33039 \\
\hline
\end{tabular}

Tuned duty cycle is the multiple of this base duty cycle, so the nodes which are in base duty cycle will be unaffected. Before sending the synchronization packet to its neighbours the sending node first checks the traffic rate which signifies the load on the node. According to the above algorithm it will modify the existing duty cycle and send the modified duty cycle to the neighbouring nodes. If the traffic on the node is high, then the duty cycle will be high of the node and it synchronizes with the other neighbours too. Without using the complex fuzzy algorithm, this regression technique will modify intelligently the duty cycle of nodes with high traffic. The high traffic signifies the occurrence of an event which must be reported to the base station without much delay.

In such model very few nodes in the whole network will be on high duty cycle during the time of communication and rest other will be able to preserve their energy while working in the normal mode of $20 \%$ or $10 \%$ duty cycle.

Mission critical applications demand quick reporting of the event to base station. So there should be an intelligent strategy to manage the nodes from one duty cycle to another.

\section{A. Analytical Model}

The paper has already discussed the strategy of tuning the duty cycle of the selected nodes in MC-MAC protocol mechanism. So in this model, we have considered the case where only selected nodes tune their duty cycle when event occurs, rest all other nodes remain unaffected. Then we have analyzed the energy performance of the proposed protocol and compared it with simple SMAC protocol.

\section{B. Assumptions}

1) There are $\rho$ nodes in the wireless sensor network deployed for monitoring in mission critical region.

2) Only $M$ nodes are tuning their duty cycle, rest other nodes are unaffected.

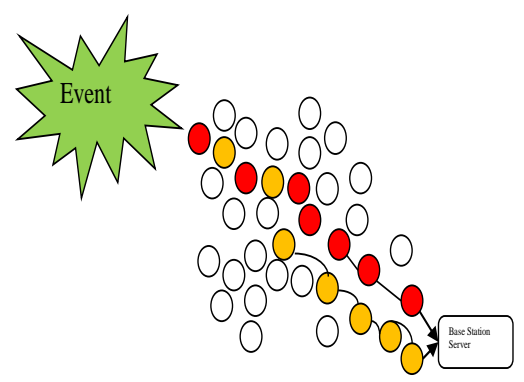

Fig. 4. Nodes with tuned duty cycle
Figure 4 shows that when an event occurs, the traffic increases on the selected nodes in an area. So to reduce the sleep delay, the duty cycle of the selected nodes is tuned based on traffic and residual energy. This reduces the sleep delay and also increases the lifetime of the critical area nodes.

Table 3 describes the notations used to for the analytical model. Here $\lambda_{10}$ considers the Time in which node considers $10 \%$ duty cycle in seconds. Another $\lambda_{20}$ considers the Time in which node considers $20 \%$ duty cycle in seconds and $\lambda_{40}$ considers the Time in which node considers $40 \%$ duty cycle in seconds.

Following equations define the parameters:

$$
\begin{aligned}
& \lambda_{10}=\mathrm{T}^{*} \boldsymbol{\mu}_{\mathbf{1}} ; \\
& \lambda_{20}=\mathrm{T}^{*} \boldsymbol{\mu}_{2} ; \\
& \lambda_{40}=\mathrm{T}^{*} \boldsymbol{\mu}_{3} ;
\end{aligned}
$$
(3).

The energy dissipation of node in active state is given as in

$E_{\text {-active }}=I_{d}+T_{r+} R_{d}$

The duty cycle of the node is given by (4).

$d=[(L / C) * 100] \rightarrow C=[(L / d) * 100$

Hence Cycle Time for $10 \%$ duty cycle is given as (5).

$C_{10}=S_{10}+L \rightarrow S_{10}=\left(C_{10}-L\right)$

Now, Energy consumed in $10 \%$ duty cycle is given by (6).

$\left.\Pi_{10}=\left(L^{*} E_{\text {_active }}\right)\right)+\left(S * S_{l}\right)$

Number of frames for $10 \%$ duty cycle in total time is given by (7).

$\alpha_{10}=\left(\lambda_{10} / C_{10}\right)$

So, in a similar way, $\alpha_{20}$ and $\alpha_{40}$ are obtained. Now Energy consumed by node during $\lambda_{10}$ when it was operating under $10 \%$ duty cycle is obtained from (8).

$E_{n 10}=\left(\Pi_{10} * \alpha_{10}\right)$

Similarly energy consumed by node in $20 \%$ and $30 \%$ duty cycle are obtained by (9) and (10).

$E_{n 20}=\left(\Pi_{20} * \alpha_{20}\right)$

$E_{n 40}=\left(\Pi_{40} * \alpha_{40}\right)$ 
TABLE. III. NOTATIONS AND THEIR MEANINGS

\begin{tabular}{|c|c|c|}
\hline S. No & Notation & Meaning \\
\hline 1. & $T$ & Total time in seconds \\
\hline 2. & $E_{n t}$ & Total energy consumed by the node in total time $\mathrm{T}$ \\
\hline 4. & $\lambda_{10}$ & Time in which node considers $10 \%$ duty cycle in seconds \\
\hline 5. & $\lambda_{20}$ & Total time which node considers $20 \%$ duty cycle in seconds \\
\hline 7. & $\mu_{1}$ & probability of the node to be in $10 \%$ duty cycle during the total time \\
\hline 8. & $\mu_{2}$ & that probability of the node to be in $20 \%$ duty cycle during the total time \\
\hline 9. & $\mu_{3}$ & that probability of the node to be in $40 \%$ duty cycle during the total time \\
\hline 10. & $\Pi_{10}$ & Energy consumed in $10 \%$ duty cycle \\
\hline 13. & $S_{l}$ & Sleep power $(15 \mu \mathrm{W})$ \\
\hline 14. & $I_{d}$ & Idle power $(14.4 \mathrm{~mW})$ for Mica Motes \\
\hline 15. & $T_{r}$ & Transmission power( $36.0 \mathrm{~mW}$ ) for Mica Motes \\
\hline 16. & $R_{d}$ & Receive power $(14.4 \mathrm{~mW})$ for Mica Motes \\
\hline 17. & $E_{\text {_active }}$ & energy dissipation of node in active state \\
\hline 18. & $d$ & Duty cycle \\
\hline 19. & 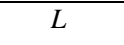 & Listen Time \\
\hline 25. & $\alpha_{40}$ & Number of frames for $40 \%$ duty cycle (frame 40 ) in total time \\
\hline 26. & $E_{n 10}$ & Energy consumed by node during $\lambda_{10}$ when it was operating under $10 \%$ duty cycle. \\
\hline 27. & $E_{n 20}$ & Energy consumed by node during $\lambda_{20}$ when it was operating under $20 \%$ duty cycle. \\
\hline 28. & $E_{n 40}$ & Energy consumed by node during $\lambda_{40}$ when it was operating under $40 \%$ duty cycle. \\
\hline 29. & $\Omega_{m}$ & Residual energy of node taking part in mission critical data transmission \\
\hline 30. & $\Omega_{t}$ & Residual energy of all mission critical nodes \\
\hline 31. & $\Omega_{n}$ & Residual energy node in $10 \%$ duty cycle \\
\hline 32. & $\Omega_{n t}$ & Residual energy of all nodes in $10 \%$ duty cycle \\
\hline 33. & $\Omega_{\text {network }}$ & Residual energy of network \\
\hline 34. & $N$ & Number of nodes in $10 \%$ duty cycle \\
\hline 35. & $M$ & Number of nodes taking part in mission critical data transmission \\
\hline
\end{tabular}


Now Total energy consumed by node in total time $\mathrm{T}$ is given by $E_{n t}=E_{n 10}+E_{n 20+} E_{n 40}$.

The residual energy of node taking part in mission critical data transmission is now given as (12).

$\Omega_{m}=\left(E_{\text {initial }}-E_{n}\right)$

So, Residual energy of all mission critical nodes is:

$\Omega_{t}=(\Omega * M)$

The value of residual energy of one node operating in $10 \%$ duty cycle for total time is obtained using the analytical model implemented in MATLAB, so $\Omega_{\mathrm{n}}=935.065 \mathrm{~J}$ and hence residual energy of all nodes in $10 \%$ duty cycle is given by (14).

$\Omega_{n t}=(935.065 * N)$

Also residual energy of network is given by:

$\Omega_{\text {network }}=\Omega_{t+} \Omega_{n t}$

From this model by changing the values of $\boldsymbol{\mu}_{1}, \boldsymbol{\mu}_{2}$ and $\boldsymbol{\mu}_{3}$ in the different traffic scenario, a remarkable improvement is shown in the residual energy of the individual node and the whole network. The values to the $\mu_{1}, \mu_{2}$ and $\mu_{3}$ are assigned initially and found the regression pattern for $\mu_{1}, \mu_{2}$ and $\mu_{3}$ also based on packet inter arrival time on the nodes. Based on this regression pattern, the values of $\mu_{1}, \mu_{2}$ and $\mu_{3}$ can be obtained and the model can be tested for any mission critical scenario.

\section{PERFORMANCE ANALYSIS}

\section{A. Input Parameters}

To analyse the performance of proposed MC-MAC protocol model we have considered high traffic scenario to low traffic scenario range as given in Tables 4 and 5 . Corresponding to that the probability value of duty cycle $\left(\boldsymbol{\mu}_{\boldsymbol{l}}\right.$, $\boldsymbol{\mu}_{2}$, and $\left.\boldsymbol{\mu}_{3}\right)$ is considered. The duty cycle values are assumed on the basis of observations obtained in analysis of SMAC protocol.

For S-MAC protocol, the duty cycle $\left(\boldsymbol{\mu}_{1}, \boldsymbol{\mu}_{2}, \boldsymbol{\mu}_{3}\right)$ are presented in Table 5. Since the duty cycle is initialized only once and is same for all the nodes during whole transmission so $\left(\boldsymbol{\mu}_{1}, \boldsymbol{\mu}_{2}, \boldsymbol{\mu}_{3}\right)$ values will be chosen as in Table 5.

Based on the regression pattern obtained in Table 5, the value of $\boldsymbol{\mu}_{\boldsymbol{I}}$ can be given as in (16).

$\boldsymbol{\mu}_{\boldsymbol{I}}=(-0.02321) *($ packets $/ \mathrm{s})+0.411847$

The value of $\boldsymbol{\mu} \boldsymbol{2}$ can be given by (17).

$\boldsymbol{\mu}_{2}=(-0.02433) *($ packets $/ \mathrm{s})+0.434356$

And the value of $\boldsymbol{\mu} 3$ can be given as in (18).

$\boldsymbol{\mu}_{3}=(0.047539) *($ packets $/ \mathrm{s})+0.153797$

With the help of these regression equations we can obtain infinite pattern of $\mu 1, \mu 2, \mu 3$ in mission critical applications and can check the energy saving performance for various cases.
TABLE. IV. INPUT PARAMETERS FOR MC-MAC PROTOCOL MODEL

\begin{tabular}{|l|l|l|l|l|}
\hline Scenarios & $\begin{array}{l}\text { Packet inter } \\
\text { arrival time in } \\
\text { seconds }\end{array}$ & $\mu 1$ & $\mu 2$ & $\mu 3$ \\
\hline Very High traffic rate & 0.1 & 0.2 & 0.2 & 0.6 \\
\hline High traffic rate & 1 & 0.2 & 0.3 & 0.5 \\
\hline Medium traffic rate & 10 & 0.2 & 0.6 & 0.2 \\
\hline Medium traffic rate & 20 & 0.4 & 0.6 & 0 \\
\hline Low traffic rate & 50 & 0.8 & 0.2 & 0 \\
\hline
\end{tabular}

TABLE. V. INPUT PARAMETERS FOR SMAC PROTOCOL MODEL

\begin{tabular}{|l|l|l|l|l|}
\hline Scenarios & $\begin{array}{l}\text { Packet } \\
\text { inter } \\
\text { arrival } \\
\text { time in } \\
\text { seconds }\end{array}$ & $\mu 1$ & $\mu 2$ & $\mu 3$ \\
\hline Very high traffic rate & 0.1 & 0 & 0 & 1 \\
\hline High traffic rate & 1 & 0 & 0 & 1 \\
\hline Medium traffic rate & 10 & 0 & 1 & 0 \\
\hline Medium traffic & 20 & 0 & 1 & 0 \\
\hline Low traffic scenario & 50 & 1 & 0 & 0 \\
\hline
\end{tabular}

\section{B. Result Analysis}

Table 6 represents the Residual energy of whole network in mission critical applications. Table 7 represents the results obtained for average residual energy of individual node in SMAC and MC-MAC protocols when they operate in mission critical area.

TABLE. VI. RESIDUAL ENERGy OF NETWORK IN MisSION CRITICAL SCENARIO

\begin{tabular}{|l|l|l|l|}
\hline $\begin{array}{l}\text { S. } \\
\text { no }\end{array}$ & $\begin{array}{l}\text { Packet inter- } \\
\text { arrival time } \\
\text { (seconds) }\end{array}$ & $\begin{array}{l}\text { Residual energy of the } \\
\text { network for MC-MAC } \\
\text { in joules }\end{array}$ & $\begin{array}{l}\text { Residual energy } \\
\text { for S-MAC in } \\
\text { joules }\end{array}$ \\
\hline 1 & 0.1 & 87805 & 48157 \\
\hline 2 & 1 & 88583 & 48157 \\
\hline 3 & 10 & 90915 & 87028 \\
\hline 4 & 20 & 92729 & 87028 \\
\hline 5 & 50 & 93247 & 93506.5 \\
\hline
\end{tabular}

TABLE. VII. AVERAGE RESIDUAL ENERGY OF NODES OPERATING IN MISSION CRITICAL SCENARIOS

\begin{tabular}{|l|l|l|l|}
\hline S.No & $\begin{array}{l}\text { Packet inter- } \\
\text { arrival time } \\
\text { (seconds) }\end{array}$ & $\begin{array}{l}\text { Residual energy of } \\
\text { single node (MC- } \\
\text { MAC) in Joules }\end{array}$ & $\begin{array}{l}\text { Residual energy of } \\
\text { single node (S- } \\
\text { MAC) in Joules }\end{array}$ \\
\hline 1 & 0.1 & 650.011 & 481.57 \\
\hline 2 & 1 & 688.882 & 481.57 \\
\hline 3 & 10 & 805.495 & 870.28 \\
\hline 4 & 20 & 896.194 & 870.28 \\
\hline 5 & 50 & 922.108 & 935.065 \\
\hline
\end{tabular}

The performance comparison of proposed MC-MAC protocol and the basic S-MAC protocol is given in Fig. 5 for the individual node and in Fig. 6 for whole network.

This analyzed the behavior of MC-MAC protocol when after an event certain selected nodes tune their duty cycle, rest are unaffected. 
In SMAC, if protocol is tuned for high duty cycle of $40 \%$ for mission critical applications, then the node always work in $40 \%$ duty cycle and consumes more energy than normal mode whereas in MC-MAC, the model considered the node to work in $40 \%$ duty cycle for maximum time (given by probability $\mu 3$ ) but not all the time when an event occurs.

The percentage energy saving in very high traffic rate scenarios (mission critical scenarios) for individual node is calculated as $16.84 \%$.In high traffic (1s), the per node energy saving is around $20.7 \%$. In medium traffic (10 packets/s), when SMAC nodes work in $20 \%$ duty cycle, in MC-MAC for some time (given by probability $\mu 3$ ) nodes work in $40 \%$ duty cycle also. Hence it is seen that SMAC saves energy.

At low traffic rates, the performance of these nodes are same as in SMAC protocol. Since the nodes saves their energy in high traffic rates so, the network lifetime increases in MCMAC protocol.

The percentage energy saving in very high traffic rate for whole network is $39.65 \%$ as shown in Fig. 6. In high traffic (1s), the energy saving for whole network it is $40.43 \%$.These results differ from other improved SMAC protocols as here the work considered the efficient data transmission as one important consideration while tuning the duty cycle. For simple applications, the performance of MC-MAC protocol is almost similar to SMAC protocol.

\section{CONCLUSION}

In this paper, we have analysed the basic SMAC protocol for residual energy, throughput and packet delivery ratio and proposed a novel approach of duty cycle adaptation based on regression pattern according to traffic. In SMAC protocol the duty cycle is fixed but the proposed MC-MAC protocol is made intelligent to dynamically change its duty cycle according to the regression formula based as a function of packet arrival rate.

The analytical model shows the behavior of the proposed mission critical MC-MAC protocol and is compared with the popular S-MAC protocol. The analytical model is based on the assumption that nodes operating in mission critical conditions will not always operate in high duty cycle. They will tune their duty cycle according to the traffic and residual energy of the nodes. The analytical results shows that the energy performance of the proposed protocol are almost improved by $40 \%$ for the whole network and by $20 \%$ for the average energy of individual node operating in mission critical environment. Since the algorithm considers the throughput, packet delivery ratio and the residual energy for obtaining the regression pattern of the duty cycle so it's different from other existing protocols. Recent proposed protocols provide the energy efficiency but at the cost of throughput and packet delivery ratio. This protocol considers the throughput and packet delivery ratio initially to calculate the duty and still saves $40 \%$ energy of the network in mission critical scenario.

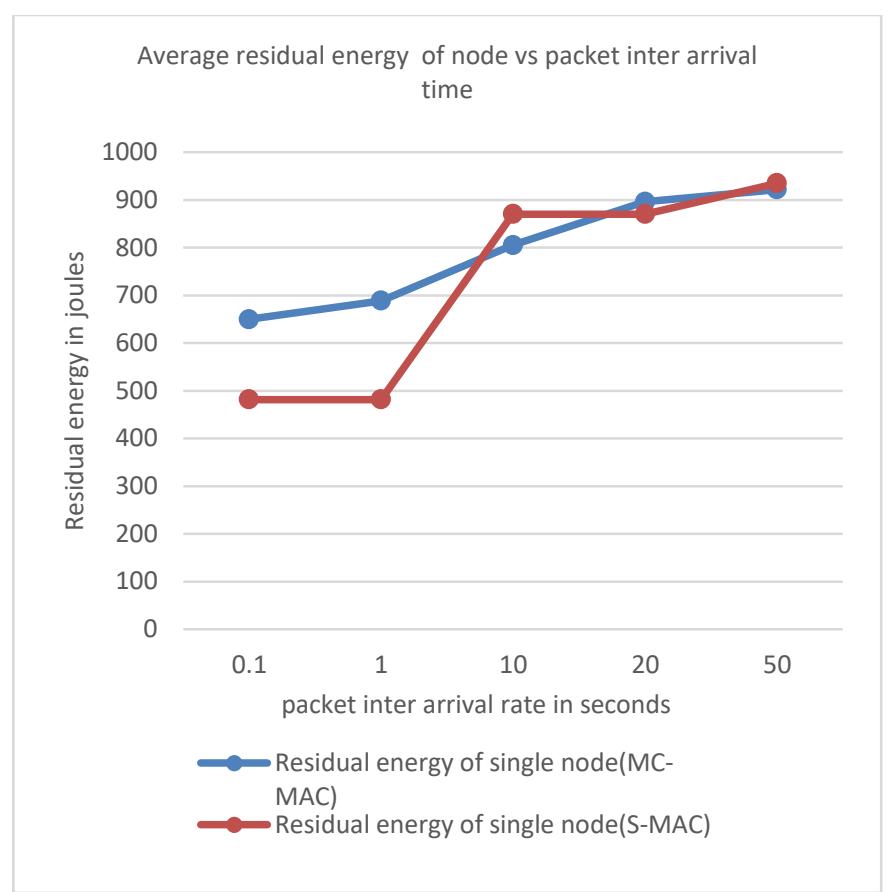

Fig. 5. Comparison of residual energy of node in MC-MAC and simple SMAC.

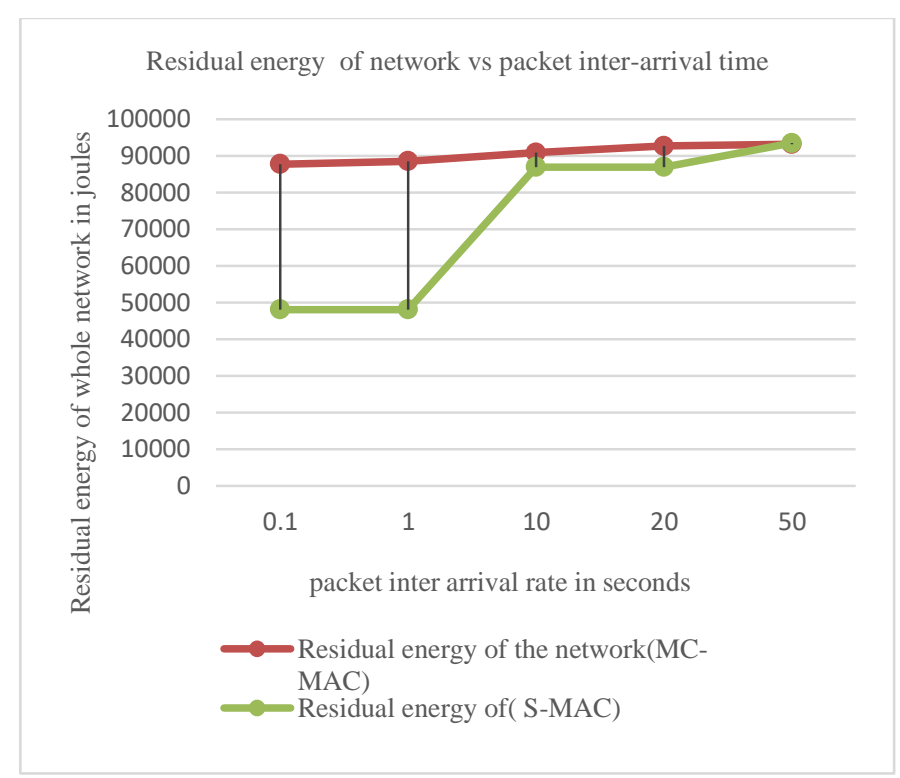

Fig. 6. Comparison of residual energy of network in MC-SMAC and simple SMAC.

Most of the proposed protocols are tested only for low traffic rates and hence the packet arrival time considered above 1 second. But this proposed protocol talks about the high traffic rates and packet inter arrival time below 1 second. So the proposed protocol is suitable for mission critical scenarios, where traffic tare suddenly become high. 


\section{FUTURE SCOPE}

The analytical model gives an idea of tremendous energy saving without deteriorating the data transmission performance of protocol. In SMAC protocol the nodes keep on sending the data without entering into sleep state after they win the contention till the end of data packets in queue of node. The sleep delay occurs only when the packets arrive at the node after the listen period. The packets wait in the queue for next listen period. In further work the proposed protocol will be implemented in ns-2 to analyse the actual energy, throughput and packet delivery ratio performance which includes this effect of not entering into sleep state when channel node has already occupied the channel for data transmission. The work will be extended to include the node selection algorithm for selecting the nodes which will send the synchronization packets to neighbouring nodes. Also the duty cycle tuning algorithm will be enhanced to include the actual load in the queue of node and its residual energy to calculate the duty cycle. This will make the MC-MAC protocol more suitable for mission critical applications by reducing the synchronization overhead and the traffic in the network in critical areas.

\section{REFERENCES}

[1] Akyildiz I. F., Su W., Sankarasubramaniam Y., Cayirci E., "A Survey on Sensor Networks," IEEE Communications Magazine, Vol. 40, Issue 8, pp. 102-114, August 2002.

[2] Ye W., Heidemann J., Estrin D., "An energy-efficient MAC protocol for wireless sensor networks," Proc. IEEE INFOCOM, New York, NY, pp. 1567-1576, June 2002.

[3] Ye W., Heidemann J., Estrin D., "Medium Access Control With Coordinated Adaptive Sleeping for Wireless Sensor Networks," IEEE/ACM Transactions on Networking, vol. 12, no. 3, pp. 493 - 506, June 2004.

[4] P. Lin, C. Qiao and X. Wang, "Medium access control with a dynamic Duty cycle for sensor networks," 2004 IEEE Wireless Communications And Networking Conference (IEEE Cat. No.04TH8733),2004, pp.15341539Vol.3.doi10.1109/WCNC.2004.1311671
[5] Shih-Hsien Yang, Hung-Wei Tseng, E. H. K. Wu and Gen-Huey Chen, "Utilization based duty cycle tuning MAC protocol for wireless sensor networks," GLOBECOM '05. IEEE Global Telecommunications Conference, 2005. St. Louis, MO, 2005, pp. 5 pp.-3262.

[6] Demirkol I., Ersoy C., Alagöz F, "MAC protocols for. Wireless Sensor Networks: a Survey," IEEE Communications Magazine, vol. 44, no. 4, pp.115-121, April 2006.

[7] Sudip Misra and Debashish Mohanta, "Adaptive listen for energyefficient medium access control in wireless sensor networks,"Multimedia Tools Appl. 47, 1 (March 2010), 121-145.

[8] Suryachai P.,Roedig U.,Scott A., "A Survey of MAC Protocols for Mission-Critical Applications in Wireless Sensor Networks", IEEE Communication Surveys \& Tutorials, vol. 14, no. 2, Second Quarter, 2012

[9] Hsu TH, Kim TH, Chen CC, Wu JS., “ A dynamic traffic-aware duty cycle adjustment MAC protocol for energy conserving in wireless sensor networks." International Journal of Distributed Sensor Networks. 2012 Feb 26.

[10] Sakya G, Sharma V, Jain PC., " Analysis of SMAC protocol for mission critical app lications in wireless sensor networks." In Advance Computing Conference (IACC), 2013 IEEE 3rd International 2013 Feb 22 ,pp. 488- 492.

[11] Sakya G, Sharma V., " Performance analysis of SMAC protocol in wireless sensor networks using network simulator (Ns-2)," In International Conference on Heterogeneous Networking for Quality, Reliability, Security and Robustness 2013 Jan 11 (pp. 42-51). Springer Berlin Heidelberg.

[12] Heejung Byun, Soogook Son, and Jungmin So. , “ Queue management based duty cycle control for end-to-end delay guarantees in wireless sensor networks.", Wirel. Netw. 19, 6 (August 2013), 1349-1360.

[13] Donghong Xu,Ke Wang, "An adaptive traffic MAC protocol based on correlation of nodes", EURASIP Journal on Wireless Communications and Networking. December 2015, 2015:258

[14] RF Monolithics Inc., http://www.rfm.com/, ASH Transceiver TR3000 Data Sheet.

[15] Gayatri Sakya, Vidushi Sharma and Trisha sawhney, "An Improved MAC Model for Critical Applications in Wireless Sensor Networks ,'International Journal of Engineering Technology, Management and Applied Sciences, Volume 4, Issue 6, ISSN 2349-4476,June 2016,pp.120-124. 